\title{
Impulsive Pinning Markovian Switching Stochastic Complex Networks with Time-Varying Delay
}

\author{
Chen Xu, ${ }^{1,2}$ Jingyi Wang, ${ }^{1}$ Jianwen Feng, ${ }^{2}$ and Yi Zhao ${ }^{2}$ \\ ${ }^{1}$ College of Information Engineering, Shenzhen University, Shenzhen 518060, China \\ ${ }^{2}$ College of Mathematics and Computational Science, Shenzhen University, Shenzhen 518060, China \\ Correspondence should be addressed to Chen Xu; xuchen_szu@szu.edu.cn
}

Received 26 April 2013; Revised 28 June 2013; Accepted 7 July 2013

Academic Editor: Wenwu Yu

Copyright (c) 2013 Chen Xu et al. This is an open access article distributed under the Creative Commons Attribution License, which permits unrestricted use, distribution, and reproduction in any medium, provided the original work is properly cited.

\begin{abstract}
The synchronization problem of stochastic complex networks with Markovian switching and time-varying delays is investigated by using impulsive pinning control scheme. The complex network possesses noise perturbations, Markovian switching, and internal and outer time-varying delays. Sufficient conditions for synchronization are obtained by employing the Lyapunov-Krasovskii functional method, Itö's formula, and the linear matrix inequality (LMI). Numerical examples are also given to demonstrate the validity of the theoretical results.
\end{abstract}

\section{Introduction and Model Description}

Collective behaviors in complex networks and systems have attracted increasing attention in recent years due to their wide applications in physics, mathematics, engineering, biology, and so forth (see $[1,2]$ and references therein). While complex networks are ubiquitously found in nature and in the modern world, such as neural networks, socially interacting animal species, power networks, wireless sensor networks, Internet, and the World Wide Web.

In the past few decades, the synchronization problems in complex networks have attracted increasing attention. Many synchronization patterns have been studied, including complete synchronization [3], cluster synchronization [4-6], phase synchronization [7], and partial synchronization [8]. There are several control methods to guide the dynamics of a complex network to a desired state, such as adaptive control [9], feedback control [10], intermittent control [11], fuzzy control [12], impulsive control [13, 14], and pinning control $[5,6,15,16]$. Synchronization of complex networks holds particular promise for applications to many fields [1721].

Synchronization in complex dynamical networks is realized via information exchanges among the interconnect nodes [22]. The signal traveling along real physical system is usually perturbed randomly by the environmental elements, such as noises, the structures of the interconnections, time delays, and the positions of nodes [9]. One popular model is the Markovian switching model driven by continuous-time Markov chains in the sciences and industries (see [23-26] and references therein). In [23, 24], Mao et al. studied stability and controllability of stochastic differential delay equations with Markovian switching, while [25, 26] discussed the exponential stability of stochastic delayed neural networks. Liu et al. [26], on the other hand, investigated the synchronization of discrete-time stochastic complex networks with Markovian jumping and mode-dependent mixed time delays. In [16], Wang et al. investigated the mean-square exponential synchronization of stochastic complex networks with Markovian switching and time-varying delays by using the pinning control method, which is described as

$$
\begin{aligned}
& d x_{i}(t) \\
& =\left\{\begin{array}{l}
f\left(t, x_{i}(t), x_{i}(t-\tau(t))\right) \\
\quad+\sum_{j=1, i \neq j}^{N} a_{i j}(r(t)) \Sigma\left(x_{j}(t)-x_{i}(t)\right)
\end{array}\right.
\end{aligned}
$$




$$
\begin{array}{r}
+\sum_{j=1, i \neq j}^{N} b_{i j}(r(t)) \Sigma\left(x_{j}\left(t-\tau_{c}(t)\right)-x_{i}\left(t-\tau_{c}(t)\right)\right) \\
\left.+u_{i}(t)\right\} d t \\
+\sigma_{i}\left(x(t), x(t-\tau(t)), x\left(t-\tau_{c}(t)\right), r(t)\right) d w_{i}(t) \\
i=1,2, \ldots, N
\end{array}
$$

where $u_{i}(t)(i=1,2, \ldots, N)$ are the linear state feedback controllers that are defined by

$$
u_{i}(t)= \begin{cases}-\varepsilon_{i}\left(x_{i}(t)-s(t)\right), & i=1,2, \ldots, l, \\ 0, & i=l+1, l+2, \ldots, N\end{cases}
$$

and $\varepsilon_{i}>0(i=1,2, \ldots, l)$ are the control gains.

Pinning control has been proved to be effective for the synchronization of complex dynamical networks with continuous state coupling $[15,16,27]$. In many systems, the impulsive effect is a common phenomenon due to instantaneous perturbations at certain moments [27, 28]. Impulsive control strategies have been widely used to stabilize and synchronize coupled complex dynamical systems, such as signal processing system, computer networks, automatic control systems, and telecommunications [13]. In [27], pinning impulsive strategy is proposed for the synchronization of stochastic dynamical networks with nonlinear coupling. Zhou et al. studied synchronization in complex delayed dynamical networks with impulsive effects in [28]. And Zhu et al., in [29], investigated the exponential stability of a class of stochastic neural networks with both Markovian jump parameters and mixed fixed time delays. Can the stochastic dynamical network with Markovian switching and timevarying delays be synchronized by impulsive pinning control? This paper is devoted to solving this problem.

In this paper, we study the synchronization of stochastic complex networks with Markovian switching by using the impulse control method. We consider a kind of stochastic complex networks with internal time-varying delayed couplings, Markovian switching, and Wiener processes. By applying the Lyapunov-Krasovskii functional method, Itö's formula and the linear matrix inequality (LMI), some sufficient conditions for synchronization of these networks are derived. Numerical examples are finally given to demonstrate the effectiveness of the proposed impulsive pinning strategy.

Notations. Throughout this paper, $\mathbb{R}^{n}$ will denote the $n$ dimensional Euclidean space and $\mathbb{R}^{n \times n}$ the set of all $n \times n$ real matrices. The superscript $T$ will denote the transpose of a matrix or a vector. And $\operatorname{Tr}(\cdot)$ stands for the trace of the corresponding matrix. $\mathbf{1}_{n}=(1,1, \ldots, 1)^{T} \in \mathbb{R}^{n}$, and $I_{n}$ is the $n$-dimensional identity matrix. For square matrices $M$, the notation $M>0(<0)$ will mean that $M$ is a positivedefinite (negative-definite) matrix. $\lambda_{\max }(A)$ and $\lambda_{\text {min }}(A)$ will denote the greatest and least eigenvalues of a symmetric matrix, respectively. $\check{p}=\max \left\{p_{1}, p_{2}, \ldots, p_{n}\right\}$, and $\widehat{p}=$ $\min \left\{p_{1}, p_{2}, \ldots, p_{n}\right\}$.

\section{Preliminaries}

Let $\left(\Omega, \mathscr{F},\left\{\mathscr{F}_{t}\right\}_{t \geq 0}, \mathscr{P}\right)$ be a complete probability space with a filtration $\left\{\mathscr{F}_{t}\right\}_{t \geq 0}$ that is right continuous with $\mathscr{F}_{0}$ containing all the $\mathscr{P}$-null sets. $C\left([-\tau, 0] ; \mathbb{R}^{n}\right)$ will denote the family of continuous functions $\phi$ from $[-\tau, 0]$ to $\mathbb{R}^{n}$ with the uniform norm $\|\phi\|^{2}=\sup _{-\tau \leq s \leq 0} \phi(s)^{T} \phi(s)$. And $C_{\mathscr{F}_{0}}^{2}\left([-\tau, 0] ; \mathbb{R}^{n}\right)$ denotes the family of all $\mathscr{F}_{0}$ measurable, $C\left([-\tau, 0] ; \mathbb{R}^{n}\right)$ valued stochastic variables $\xi=\{\xi(\theta):-\tau \leq \theta \leq 0\}$ such that $\int_{-\tau}^{0} \mathbb{E}\|\xi(s)\|^{2} d s \leq \infty$, where $\mathbb{E}$ stands for the correspondent expectation operator with respect to the given probability measure $\mathscr{P}$.

Consider a complex network consisting of $N$ identical nodes with Markovian switching

$$
\begin{aligned}
d x_{i}(t)=\left\{f\left(x_{i}(t), x_{i}(t-\tau(t))\right)\right. & \\
& +\sum_{j=1}^{N} a_{i j}^{[r(t)]} \sum x_{j}(t) \\
& \left.+\sum_{j=1}^{N} b_{i j}^{[r(t)]} \sum x_{j}\left(t-\tau_{c}(t)\right)\right\} d t \\
+ & \sigma_{i}^{[r(t)]}\left(x(t), x(t-\tau(t)), x\left(t-\tau_{c}(t)\right)\right) d w(t), \quad i=1,2, \ldots, N,
\end{aligned}
$$

where $x_{i}(t)=\left(x_{i 1}(t), x_{i 2}(t), \ldots, x_{i n}(t)\right)^{T} \in \mathbb{R}^{n}$ is the state vector of the $i$ th node of the network, $f\left(x_{i}(t), x_{i}(t-\tau(t))\right)=$ $\left[f_{1}\left(x_{i}(t), x_{i}(t-\tau(t))\right), f_{2}\left(x_{i}(t), x_{i}(t-\tau(t))\right), \ldots, f_{n}\left(x_{i}(t), x_{i}(t-\right.\right.$ $\tau(t)))]^{T}$ is a continuous vector-valued function, $\Sigma=\operatorname{diag}\left\{\varrho_{1}\right.$, $\left.\varrho_{2}, \ldots, \varrho_{n}\right\}$ is an inner coupling matrix of the networks that satisfies $\varrho_{j}>0, j=1,2, \ldots, n$, and $r(t)$ are the continuoustime Markov processes that describe the evolution of the nodes at time $t$. Here, $A^{[r(t)]}=\left[a_{i j}^{[r(t)]}\right] \in \mathbb{R}^{n \times n}$ and $B^{[r(t)]}=$ $\left[b_{i j}^{[r(t)]}\right] \in \mathbb{R}^{n \times n}$ are the outer coupling matrices of the network at time $t$ at nodes $r(t), t-\tau_{c}(t)$, respectively, such that $a_{i j}^{[r(t)]} \geq 0$ for $i \neq j, a_{i i}^{[r(t)]}=-\sum_{j=i, j \neq i}^{N} a_{i j}^{[r(t)]}, b_{i j}^{[r(t)]} \geq 0$ for $i \neq j$ and $b_{i i}^{[r(t)]}=-\sum_{j=i, j \neq i}^{N} b_{i j}^{[r(t)]}$. Figure 1 shows the topology structures of the switching networks for 5 nodes. $\tau(t)$ is the inner time-varying delay satisfying $\tau \geq \tau(t) \geq 0$ and $\tau_{c}(t)$ is the coupling time-varying delay satisfying $\tau_{c} \geq$ $\tau_{c}(t) \geq 0$. Finally, $\sigma_{i}^{[r(t)]}\left(x(t), x(t-\tau(t)), x\left(t-\tau_{c}(t)\right)\right)=$ $\sigma_{i}^{[r(t)]}\left(x_{1}(t), \ldots, x_{n}(t), x_{1}(t-\tau(t)), \ldots, x_{n}(t-\tau(t)), x_{1}(t-\right.$ $\left.\left.\tau_{c}(t)\right), \ldots, x_{n}\left(t-\tau_{c}(t)\right)\right) \in \mathbb{R}^{n \times n}$ and $w(t)=\left(w_{1}(t)\right.$, $\left.w_{2}(t), \ldots, w_{n}(t)\right)^{T} \in \mathbb{R}^{n}$ is a bounded vector-form Weiner process, satisfying

$$
\begin{gathered}
\mathbb{E} w_{j}(t)=0, \quad \mathbb{E} w_{j}^{2}(t)=1, \\
\mathbb{E} w_{j}(t) w_{j}(s)=0 \quad(s \neq t) .
\end{gathered}
$$




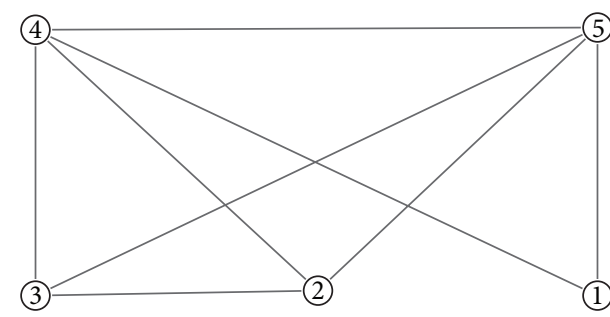

(A1)

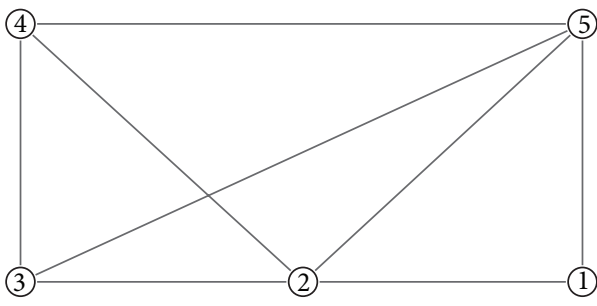

(A2)

(a)

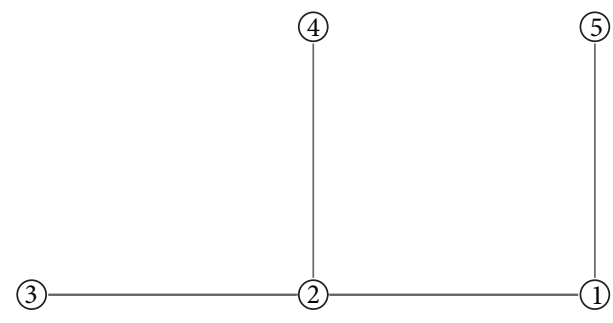

(B1)

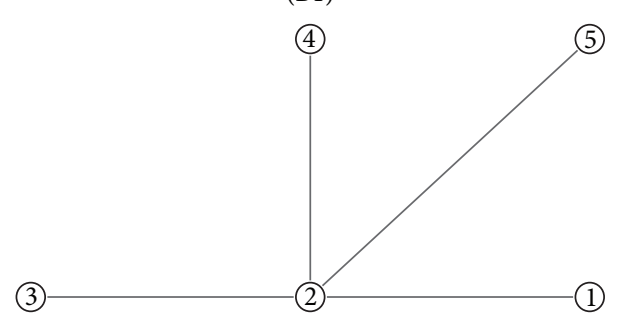

(B2)

(b)

FIGURE 1: The topology structures of the switching networks for 5 nodes; (A1) and (A2) the topology structures of the coupling matrix $A^{1}$ and $A^{2}$, respectively; (B1) and (B2) the topology structures of the coupling matrix $B^{1}$ and $B^{2}$, respectively.

Let $r(t), t>0$ be a right-continuous Markov chain on a probability space that takes values in a finite state space $S=$ $1,2, \ldots, M$ with a generator $\Gamma=\left[\gamma_{i j}\right] \in \mathbb{R}^{M \times M}$ given by

$$
P\{r(t+\Delta)=j \mid r(t)=i\}= \begin{cases}\gamma_{i j} \Delta+o(\Delta) & \text { if } i \neq j \\ 1+\gamma_{i i} \Delta+o(\Delta) & \text { if } i=j\end{cases}
$$

for some $\Delta>0$. Here $\gamma_{i j} \geq 0$ is the transition rate from $i$ to $j$ if $i \neq j$ and $\gamma_{i i}=-\sum_{i \neq j} \gamma_{i j}$, In this paper, $A^{[r]}$ is assumed to be symmetric and irreducible, and $B^{[r]}$ is assumed to be symmetric, for $r=1,2, \ldots, M$.

The initial conditions associated with (3) are

$$
x_{i}(s)=\xi_{i}(s), \quad-\check{\tau} \leq s \leq 0, \quad i=1,2, \ldots, N,
$$

where $\check{\tau}=\max \left\{\tau(t), \tau_{c}(t)\right\}, \xi_{i} \in C_{\mathscr{F}_{0}}^{b}\left([-\check{\tau}, 0], \mathbb{R}^{n}\right)$ with the norm $\left\|\xi_{i}\right\|^{2}=\sup _{-\check{\tau} \leq s \leq 0} \xi_{i}(s)^{T} \xi_{i}(s)$.

The impulse controllers are defined by

$$
\begin{gathered}
\Delta x_{i}\left(t_{k}\right)=x_{i}\left(t_{k}^{+}\right)-x_{i}\left(t_{k}^{-}\right)=\epsilon_{i k} x_{i}\left(t_{k}^{-}\right), \\
t=t_{k}, \quad k \in Z^{+}, \quad i=1,2, \ldots, N,
\end{gathered}
$$

where $\epsilon_{\mathrm{i} k}$ are constants, and $\left\{t_{1}, t_{2}, t_{3}, \ldots\right\}$ is the impulsive sequence of strictly increasing impulsive instants satisfying $\lim _{k \rightarrow \infty} t_{k}=+\infty$, and $t_{k}-t_{k-1}=T$ for $k>1$.

In the case that system (3) reaches synchronization, that is, $x_{1}(t)=x_{2}(t)=\cdots=x_{N}(t)=s(t)$, we have the following synchronized state equation:

$$
\begin{aligned}
d s(t)= & f(s(t), s(t-\tau(t))) d t \\
& +\sigma(s(t), s(t-\tau(t))) d w(t) .
\end{aligned}
$$

In the paper, we would control the system (3) to the desired trajectory $s(t)$. Define $e_{i}(t)=x_{i}(t)-s(t)(i=1$, $2, \ldots, N)$ as the synchronization error. Then, according to the controller (7), the error system is

$$
\begin{gathered}
d e_{i}(t)=\left\{f\left(x_{i}(t), x_{i}(t-\tau(t))\right)-f(s(t), s(t-\tau(t)))\right. \\
\left.+\sum_{j=1}^{N} a_{i j}^{[r(t)]} \sum e_{j}(t)+\sum_{j=1}^{N} b_{i j}^{[r(t)]} \sum e_{j}\left(t-\tau_{c}(t)\right)\right\} d t \\
+\sigma_{i}^{[r(t)]}\left(e(t), e(t-\tau(t)), e\left(t-\tau_{c}(t)\right)\right) d w(t), \\
t \neq t_{k}, \quad k \in Z^{+}, \quad i=1,2, \ldots, N,
\end{gathered}
$$$$
\Delta e_{i}\left(t_{k}\right)=\epsilon_{i k} e_{i}\left(t_{k}^{-}\right), \quad t=t_{k}, \quad k \in Z^{+}, \quad i=1,2, \ldots, N,
$$

where $\sigma_{i}^{[r(t)]}\left(e(t), e(t-\tau(t)), e\left(t-\tau_{c}(t)\right)\right)=\sigma_{i}^{[r(t)]}(x(t), x(t-$ $\left.\tau(t)), x\left(t-\tau_{c}(t)\right)\right)-\sigma(s(t), s(t-\tau(t)))$.

Definition 1 (see $[16,27]$ ). The complex network (3) is said to be exponentially synchronized in mean square if the trivial solution of system (9) is such that

$$
\sum_{i=1}^{N} \mathbb{E}\left\|e_{i}\left(t, t_{0}, \xi_{i}\right)\right\|^{2} \leq K e^{-\kappa t},
$$

for some $K>0$ and $\kappa>0$ under any initial data $\xi_{i} \epsilon$ $\mathscr{C}_{\mathscr{F}_{0}}^{b}\left([-\check{\tau}, 0] ; \mathbb{R}^{n}\right)$

Definition 2 (see $[9,11,16])$. A continuous function $f(x, y)$ : $\mathbb{R}^{n} \times \mathbb{R}^{n} \rightarrow \mathbb{R}^{n}$ is said to belong to the function class QUAD, 
denoted by $f \in \operatorname{QUAD}(P, \Delta, \eta, \theta)$ for some given matrix $\Sigma=\operatorname{diag}\left\{\varrho_{1}, \varrho_{2}, \ldots, \varrho_{n}\right\}$ if there exists a positive definite diagonal matrix $P=\operatorname{diag}\left\{p_{1}, p_{2}, \ldots, p_{n}\right\}$, a diagonal matrix $\Delta=\operatorname{diag}\left\{\delta_{1}, \delta_{2}, \ldots, \delta_{n}\right\}$ and constants $\eta>0, \theta>0$ such that $f(\cdot)$ satisfies the condition

$$
\begin{aligned}
& (x-y)^{T} P((f(x, z)-f(y, w))-\Delta \Sigma(x-y)) \\
& \leq-\eta(x-y)^{T}(x-y)+\theta(z-w)^{T}(z-w)
\end{aligned}
$$

for all $x, y, z, w \in \mathbb{R}^{n}$.

The following assumptions are usual and will be used throughout this paper for establishing the synchronization conditions $[9,11,16]$.

(H1) $\tau(t)$ and $\tau_{c}(t)$ are bounded and continuously differentiable functions such that $0<\tau(t) \leq \tau, \dot{\tau}(t)<\bar{\tau}<1$, $0<\tau_{c}(t) \leq \tau_{c}$ and $\dot{\tau}_{c}(t)<\bar{\tau}_{c}<1$. Let $\check{\bar{\tau}}=\max \left\{\bar{\tau}, \bar{\tau}_{c}\right\}$.

(H2) There exist positive definite constant matrices $Y_{i 1}^{[r]}$, $\Upsilon_{i 2}^{[r]}$ and $\Upsilon_{i 3}^{[r]}$ for $i=1,2, \ldots, N$ and $r=1,2, \ldots, M$ such that

$$
\begin{aligned}
& \operatorname{Tr}\left[\sigma_{i}^{[r]}\left(e(t), e(t-\tau(t)), e\left(t-\tau_{c}(t)\right)\right)^{T}\right. \\
& \left.\quad \times \sigma_{i}^{[r]}\left(e(t), e(t-\tau(t)), e\left(t-\tau_{c}(t)\right)\right)\right] \\
& \leq \sum_{j=1}^{N} e_{j}(t)^{T} \Upsilon_{i 1}^{[r]} e_{j}(t) \\
& \quad+\sum_{j=1}^{N} e_{j}(t-\tau(t))^{T} \Upsilon_{i 2}^{[r]} e_{j}(t-\tau(t)) \\
& +\sum_{j=1}^{N} e_{j}\left(t-\tau_{c}(t)\right)^{T} \Upsilon_{i 3}^{[r]} e_{j}\left(t-\tau_{c}(t)\right) .
\end{aligned}
$$

Remark 3. Considering Definition 2 and assumption (H2), there exists a unique solution of (9) under the initial data $\xi_{i} \in \mathscr{C}_{\mathscr{F}_{0}}^{b}\left([-\tau, 0] ; \mathbb{R}^{n}\right)($ see $[23,24])$.

Lemma 4 (see $[23,24])$. Consider a stochastic delayed differential equation with Markovian switching of the form

$$
\begin{aligned}
d x(t)= & f(x(t), x(t-\tau), r(t)) d t \\
& +\sigma(x(t), x(t-\tau), r(t)) d \omega(t)
\end{aligned}
$$

on $t \geq 0$ with initial value $x_{0}=\xi \in C_{F_{0}}^{b}\left([-\tau, 0] ; \mathbb{R}^{n}\right)$, where

$$
f: \mathbb{R}^{n} \times \mathbb{R}^{n} \times S \rightarrow \mathbb{R}^{n}, \quad \sigma: \mathbb{R}^{n} \times \mathbb{R}^{n} \times S \rightarrow \mathbb{R}^{n \times m}
$$

Let $C^{2,1}\left(\mathbb{R}_{+} \times \mathbb{R}^{n} ; \mathbb{R}_{+}\right)$be the family of all the nonnegative functions $V(t, x, r)$ on $\mathbb{R}_{+} \times \mathbb{R}^{n} \times S$ that are twice continuously differentiable in $x$ and once differentiable in $t$. Let $V \in$
$C^{2,1}\left(\mathbb{R}_{+} \times \mathbb{R}^{n} \times S ; \mathbb{R}_{+}\right)$. Define an operator $\mathscr{L} V$ from $\mathbb{R}^{n} \times \mathbb{R}_{+} \times S$ to $\mathbb{R}^{n}$ by

$$
\begin{aligned}
\mathscr{L} V(t, x, r)= & V_{t}(t, x, r)+V_{x}(t, x, r) f(x, r) \\
& +\frac{1}{2} \operatorname{Tr}\left[\sigma(x, r)^{T} V_{x x} \sigma(x, r)\right] \\
& +\sum_{j=1}^{M} \gamma_{i j} V(t, x, j),
\end{aligned}
$$

where $V_{t}(t, x, r)=\partial V(t, x, r) / \partial t, V_{x}(t, x, r)=(\partial V(t, x$, $\left.r) / \partial x_{1}, \ldots, \partial V(t, x, r) / \partial x_{n}\right)$, and $V_{x x}(t, x, r)=\left(\partial^{2} V(t, x\right.$, $\left.r) / \partial x_{i} x_{j}\right)_{n \times n}$. If $V \in C^{2,1}\left(\mathbb{R}_{+} \times \mathbb{R}^{n} \times S ; \mathbb{R}_{+}\right)$, and then

$$
\mathbb{E} V(t, x(t), r)=\mathbb{E} V\left(t_{0}, x\left(t_{0}\right), r\right)+\mathbb{E} \int_{t_{0}}^{t} \mathscr{L} V(s, x(s), r) d s
$$

for all $\infty>t>t_{0} \geq 0$, as long as the expectations of the integrals exist.

\section{Main Result}

In this section, we will deduce our main results.

Theorem 5. Let assumptions (H1) and (H2) be true and let $f \in \operatorname{QUAD}(P, \Delta, \eta, \theta)$. If there exist positive constants $\alpha_{r}$ and $\beta_{r}$ such that

$$
\begin{gathered}
{\left[\begin{array}{cc}
A^{[r]}+\check{\delta} I_{N}-\alpha_{r} I_{N} & \frac{B^{[r]}}{2} \\
\frac{B^{[r]}}{2} & -\beta_{r} I_{N}
\end{array}\right] \leq 0, \quad \text { for } r=1,2, \ldots, M,} \\
\check{\tau} \leq \theta T, \quad \check{\tau} \leq(1-\theta) T, \quad 0 \leq \check{\bar{\tau}} \leq 1-\frac{\check{q}(\check{b}+\check{c})}{1+\theta}, \\
\varphi(\check{\tau}+T)+2 \ln \frac{\check{q}}{\widehat{q}}\left|1+\epsilon_{v}\right|-\gamma T<0, \\
\left(\frac{1}{b_{1}+c_{1}}, \frac{1}{b_{2}+c_{2}}, \ldots, \frac{1}{b_{M}+c_{M}}\right)^{T}>\widetilde{\Gamma}^{-1} \mathbf{1}_{M},
\end{gathered}
$$

where

$$
\begin{gathered}
\varphi=1+\theta+\gamma \check{q}+\frac{\check{b} \check{q}}{1-\bar{\tau}} e^{\gamma \tau}+\frac{\check{c} \check{q}}{1-\bar{\tau}_{c}} e^{\gamma \tau_{c}}, \\
\widetilde{\Gamma}=\operatorname{diag}\left\{a_{1}, a_{2}, \ldots, a_{M}\right\}+\Gamma \\
\check{a}=\max _{r \in S} a_{r}, \quad a_{r}=\frac{\lambda_{\max }\left(-2 \eta I_{n}+\check{p} \sum_{i=1}^{N} \Upsilon_{i 1}^{[r]}+2 \alpha_{r} P \Sigma\right)}{\check{p}}, \\
\check{b}=\max _{r \in S} b_{r}, \quad b_{r}=\frac{\lambda_{\max }\left(\sum_{i=1}^{N} P \Upsilon_{i 2}^{[r]}+2 \theta I_{N}\right)}{\widehat{p}}, \\
\check{c}=\max _{r \in S} c_{r}, \quad c_{r}=\frac{\lambda_{\max }\left(\sum_{i=1}^{N} P Y_{i 3}^{[r]}+2 \beta_{r} P \Sigma\right)}{\widehat{p}},
\end{gathered}
$$


then the solutions $e_{1}(t), e_{2}(t), \ldots$, and $e_{N}(t)$ of system (9) are exponentially stable in mean square. It means that the complex dynamical network (3) can be exponentially controlled to the objective trajectory $s(t)$ under the controllers (7).

Proof. By (44), there exists a sufficiently small $\operatorname{constant} \theta>0$ such that

$$
\left(\frac{1}{b_{1}+c_{1}}, \frac{1}{b_{2}+c_{2}}, \ldots, \frac{1}{b_{M}+c_{M}}\right)^{T} \geq(1+\theta) \widetilde{\Gamma}^{-1} \mathbf{1} .
$$

Set $(1+\theta) \widetilde{\Gamma}^{-1} \mathbf{1}=q=\left(q_{1}, q_{2}, \ldots, q_{M}\right)^{T}$. Then $\widetilde{\Gamma} q=(1+\theta) \mathbf{1}_{M}$, that is,

$$
\left(b_{r}+c_{r}\right) q_{r} \leq 1, \quad a_{r} q_{r}+\sum_{s=1}^{M} \gamma_{r s} q_{s}=1+\theta .
$$

For $1 \leq r \leq M$, define the Lyapunov-Krasovskii function

$$
V(e(t), r)=q_{r} \frac{1}{2} \sum_{i=1}^{N} e_{i}(t)^{T} P e_{i}(t),
$$

and let $\vec{e}^{k}(t)=\left(e_{1 k}(t), e_{2 k}(t), \ldots, e_{N k}(t)\right)^{T}, k=1,2, \ldots, n$. For any $t \in\left(t_{k-1}, t_{k}\right], k=1,2, \ldots$, by Lemma 4 , we have

$$
\begin{aligned}
& \mathscr{L} V(e(t), r) \\
& =q_{r} \sum_{i=1}^{N} e_{i}(t)^{T} P\left\{f\left(x_{i}(t), x_{i}(t-\tau(t))\right)\right. \\
& -f(s(t), s(t-\tau(t)))+\sum_{j=1}^{N} a_{i j}^{[r]} \Sigma e_{j}(t) \\
& \left.+\sum_{j=1}^{N} b_{i j}^{[r]} \Sigma e_{j}\left(t-\tau_{c}(t)\right)\right\} \\
& +\frac{1}{2} q_{r} \sum_{i=1}^{N} \operatorname{Tr}\left\{\sigma_{i}\left(x(t), x(t-\tau(t)), x\left(t-\tau_{c}(t)\right), r\right)^{T}\right. \\
& \left.\times P \sigma_{i}\left(x(t), x(t-\tau(t)), x\left(t-\tau_{c}(t)\right), r\right)\right\} \\
& +\sum_{s=1}^{M} \gamma_{r s} q_{s} \frac{1}{2} \sum_{i=1}^{N} e_{i}(t)^{T} P e_{i}(t) \\
& \leq q_{r}\left\{-\eta \sum_{i=1}^{N} e_{i}(t)^{T} e_{i}(t)+\theta \sum_{i=1}^{N} e_{i}(t-\tau(t))^{T} e_{i}(t-\tau(t))\right. \\
& +\sum_{k=1}^{n} p_{k} \varrho_{k} \delta_{k} \widetilde{e}^{-k}(t)^{T} \widetilde{e}^{k}(t)+\sum_{k=1}^{n} p_{k} \varrho_{k} \widetilde{e}^{-k}(t)^{T} A^{[r]} \widetilde{e}^{k}(t) \\
& +\sum_{k=1}^{n} p_{k} \varrho_{k} \widetilde{e}^{k}(t)^{T} B^{[r]} \widetilde{e}^{k}\left(t-\tau_{c}(t)\right) \\
& +\frac{1}{2} \check{p} \sum_{j=1}^{N}\left[\sum_{i=1}^{N} e_{i}(t)^{T} \Upsilon_{j 1}^{[r]} e_{i}(t)\right. \\
& +\sum_{i=1}^{N} e_{i}(t-\tau(t))^{T} \Upsilon_{j 2}^{[r]} e_{i}(t-\tau(t)) \\
& \left.\left.+\sum_{i=1}^{N} e_{i}\left(t-\tau_{c}(t)\right)^{T} \Upsilon_{j 3}^{[r]} e_{i}\left(t-\tau_{c}(t)\right)\right]\right\}
\end{aligned}
$$

$$
\begin{aligned}
& +\sum_{s=1}^{M} \gamma_{r s} q_{s} \frac{1}{2} \sum_{i=1}^{N} e_{i}(t)^{T} P e_{i}(t) \\
& =q_{r}\left\{\sum_{i=1}^{N} e_{i}(t)^{T}\left(-\eta I_{N}+\frac{1}{2} \check{p} \sum_{j=1}^{N} \Upsilon_{j 1}^{[r]}+\alpha_{r} P \Sigma\right) e_{i}(t)\right. \\
& +\sum_{i=1}^{N} e_{i}(t-\tau(t))^{T}\left(\theta+\frac{1}{2} \check{p} \sum_{j=1}^{N} \Upsilon_{j 2}^{[r]}\right) e_{i}(t-\tau(t)) \\
& \left.+\sum_{i=1}^{N} e_{i}\left(t-\tau_{c}(t)\right)^{T}\left(\frac{1}{2} \tilde{p} \sum_{j=1}^{N} \Upsilon_{j 3}^{[r]}+\beta_{r} P \Sigma\right) e_{i}\left(t-\tau_{c}(t)\right)\right\} \\
& +\sum_{s=1}^{M} \gamma_{r s} q_{s} \frac{1}{2} \sum_{i=1}^{N} e_{i}(t)^{T} P e_{i}(t) \\
& +q_{r}\left\{\sum_{k=1}^{n} p_{k} e_{k} e^{-k}(t)^{T}\left[A^{[r]}+\left(\check{\delta}-\alpha_{r}\right) I_{N}\right] \tilde{e}^{k}(t)\right. \\
& +\sum_{k=1}^{n} p_{k} e_{k} e^{\widetilde{e}^{k}}(t)^{T} B^{[r]} \tilde{e}^{k}\left(t-\tau_{c}(t)\right) \\
& \left.\quad-\sum_{k=1}^{n} p_{k} e_{k} e^{\tilde{e}^{k}}\left(t-\tau_{c}(t)\right)^{T} \beta_{r} \tilde{e}^{k k}\left(t-\tau_{c}(t)\right)\right\} \\
& +\sum_{s=1}^{M} \gamma_{r s} q_{s} \frac{1}{2} \sum_{i=1}^{N} e_{i}(t)^{T} P e_{i}(t) . \\
& \quad+q_{r}\left\{\sum_{i=1}^{N} e_{i}(t)^{T}\left(-\eta I_{N}+\frac{1}{2} \check{p} \sum_{j=1}^{N} \Upsilon_{j 1}^{[r]}+\alpha_{r} P \Sigma\right) e_{i}(t)\right. \\
& +\sum_{i=1}^{N} e_{i}(t-\tau(t))^{T}\left(\theta I_{N}+\frac{1}{2} \check{p} \sum_{j=1}^{N} \Upsilon_{j 2}^{[r]}\right) e_{i}(t-\tau(t)) \\
& \left.\left.+\tau_{c}(t)\right)^{T}\left(\frac{1}{2} \check{p} \sum_{j=1}^{N} \Upsilon_{j 3}^{[r]}+\beta_{r} P \Sigma\right) e_{i}\left(t-\tau_{c}(t)\right)\right\}
\end{aligned}
$$

Let

$$
E(t)=\frac{1}{2} \sum_{i=1}^{N} e_{i}(t)^{T} P e_{i}(t)
$$

then we have

$$
\begin{aligned}
\mathscr{L} V(t) \leq & a_{r} q_{r} E(t)+b_{r} q_{r} E(t-\tau(t)) \\
& +c_{r} q_{r} E\left(t-\tau_{c}(t)\right)+\sum_{s=1}^{M} \gamma_{r s} q_{s} E(t),
\end{aligned}
$$

and by (20), we have

$$
\mathscr{L} V(t) \leq(1+\theta) E(t)+\check{b} \check{q} E(t-\tau(t))+\check{c} \check{q} E\left(t-\tau_{c}(t)\right) .
$$

Define

$$
W(t)=e^{\gamma t} V(t) .
$$


Use (25) to compute the operator

$$
\begin{aligned}
\mathscr{L} W(t) & =e^{\gamma t}[\gamma V(t)+\mathscr{L} V(t)] \\
\leq e^{\gamma t}[\gamma \check{q} E(t)+(1+\theta) E(t) & \\
& \left.+\check{b} \check{q} E(t-\tau(t))+\check{c} \check{q} E\left(t-\tau_{c}(t)\right)\right] .
\end{aligned}
$$

The generalized Itô's formula gives

$$
e^{\gamma t} \mathbb{E} V(t)=e^{\gamma t_{0}} \mathbb{E} V\left(t_{0}\right)+\mathbb{E} \int_{t_{0}}^{t} \mathscr{L} W(s) d s,
$$

for any $t_{k}>t>t_{0}>t_{k-1} \geq 0$. Hence we have

$$
\begin{aligned}
e^{\gamma t} \mathbb{E} V(t) \leq & e^{\gamma t_{0}} \mathbb{E} V\left(t_{0}\right) \\
& +\mathbb{E} \int_{t_{0}}^{t} e^{\gamma s}[\gamma \check{q} E(s)+(1+\theta) E(s) \\
& +\check{b} \check{q} E(s-\tau(s)) \\
& \left.+\check{c} \check{q} E\left(s-\tau_{c}(s)\right)\right] d s \\
\leq & \check{q} e^{\gamma t_{0}} \mathbb{E} E\left(t_{0}\right)+(\gamma \check{q}+1+\theta) \\
& \times \int_{t_{0}}^{t} e^{\gamma s} \mathbb{E} E(s) d s \\
& +\check{b} \check{q} e^{\gamma \tau} \int_{t_{0}}^{t} e^{\gamma(s-\tau(s))} \mathbb{E} E(s-\tau(s)) d s \\
& +\check{c} \check{q} e^{\gamma \tau_{c}} \int_{t_{0}}^{t} e^{\gamma\left(s-\tau_{c}(s)\right)} \mathbb{E} E\left(s-\tau_{c}(s)\right) d s .
\end{aligned}
$$

By changing variable $s-\tau(s)=u$, we have

$$
\begin{aligned}
\int_{t_{0}}^{t} e^{\gamma(s-\tau(s))} \mathbb{E} E(s-\tau(s)) d s \\
\quad=\int_{t_{0}-\tau\left(t_{0}\right)}^{t-\tau(t)} e^{\gamma u} \mathbb{E} E(u) \frac{d u}{1-\dot{\tau}(t)} \\
\quad \leq \frac{1}{1-\bar{\tau}} \int_{t_{0}-\tau}^{t} e^{\gamma u} \mathbb{E} E(u) d u .
\end{aligned}
$$

Similarly, we have

$$
\int_{t_{0}}^{t} e^{\gamma\left(s-\tau_{c}(s)\right)} \mathbb{E} E(s-\tau(s)) d s \leq \frac{1}{1-\bar{\tau}_{c}} \int_{t_{0}-\tau_{c}}^{t} e^{\gamma u} \mathbb{E} E(u) d u .
$$

Substituting (30) and (31) into (29), we get

$$
e^{\gamma t} \mathbb{E} V(t) \leq q_{r} e^{\gamma t_{0}} \mathbb{E} V\left(t_{0}\right)+\varphi \int_{t_{0}-\check{\tau}}^{t} e^{\gamma u} \mathbb{E} E(u) d u
$$

By using Gronwall inequality, we have

$$
\mathbb{E} E(t) \leq \frac{\check{q}}{\widehat{q}} \mathbb{E} E\left(t_{0}\right) e^{\varphi\left(t-t_{0}+\check{\tau}\right)+\gamma\left(t_{0}-t\right)} .
$$

On the other hand, from the construction of $E(t)$, we have

$$
E\left(t_{k}\right) \leq\left(1+\epsilon_{k}\right)^{2} E\left(t_{k}^{-}\right),
$$

where $\left|1+\epsilon_{k}\right|=\max _{i=1,2, \ldots, N}\left|1+\epsilon_{i k}\right|$.

According to (33)-(34), let $k=\left\lfloor\left(t-t_{0}\right) / T\right\rfloor$, for any $t \in$ $\left[t_{k-1}, t_{k}\right)$, and we get

$$
\begin{aligned}
& \mathbb{E} V(t) \leq \frac{\check{q}}{\hat{q}} \mathbb{E} E\left(t_{k}\right) e^{\varphi\left(t-t_{k}+\check{\tau}\right)+\gamma\left(t_{k}-t\right)} \\
& \leq \frac{\check{q}}{\hat{q}} \mathbb{E} V\left(t_{k-1}^{-}\right) e^{\varphi\left(t-t_{k-1}+\check{\tau}\right)+\gamma\left(t_{k}-t\right)+2 \ln \left|1+\epsilon_{k-1}\right|} \\
& \leq \cdots \leq\left(\frac{\check{q}}{\hat{q}}\right)^{k-1} \mathbb{E} E(0) e^{\varphi(t+k \check{\tau})-\gamma t+\sum_{v=1}^{k-1} 2 \ln \left|1+\epsilon_{v}\right|} . \\
& \text { Let }|1+\epsilon|=\max _{v \in Z^{+}}\left|1+\epsilon_{v}\right|, \text { and we have } \\
& \mathbb{E} E(t) \leq \mathbb{E} E(0) e^{\varphi(t+k \check{\tau})-\gamma t+2(k-1) \ln (\check{q} /(\widehat{q})|1+\epsilon|} .
\end{aligned}
$$

Using condition (43) of Theorem 5, there exist a number $\eta$ such that $\mathbb{E} E(t) \leq \mathbb{E} E\left(t_{0}\right) e^{-\eta t}$. Hence, $\mathbb{E}\left\|e_{i}(t)\right\| \leq\left(E\left(t_{0}\right) /\right.$ $\check{p})^{1 / 2} e^{-(\eta / 2)\left(t-t_{0}\right)}$. The proof of Theorem 5 is completed.

Remark 6. The stochastic networks studied before are without topological switch, and the time delays are always assumed to be fixed. However, for the sake of applications in the real work, these two points above should be taken into consideration. Of course, it will enhance the difficulties of the investigations on this network. For example, if the network has Markovian switching topology, the structure of the network is fast varying and the Lyapunov function is hard to be determined. By using the Lyapunov-Krasovskii functional, Itö's formula, and LMI, the exponential stability criterion of the pinning impulsive controlled Markovian switching stochastic dynamical network with time-varying delays was obtained. This also showed that the impulsive pinning control is a kind of cheap control strategy for guiding complex dynamical networks to the objective trajectory.

To make Theorem 5 more applicative, we give the following corollaries.

When complex dynamic networks (3) are considered without coupled delay time $\left(B^{[r(t)]}=B\right)$, we can get the following corollary.

Corollary 7. Let assumptions (H1) and (H2) be true and let $f \in \operatorname{QUAD}(P, \Delta, \eta, \theta)$. If there exist positive constants $\alpha_{r}$ and $\beta_{r}$ such that

$$
\begin{gathered}
A^{[r]}+\check{\delta} I_{N}-\alpha_{r} I_{N} \leq 0, \quad \text { for } r=1,2, \ldots, M, \\
\tau \leq \theta T, \quad \tau \leq(1-\theta) T, \quad 0 \leq \bar{\tau} \leq 1-\frac{\check{q} \breve{b}}{1+\theta}, \\
\varphi(\check{\tau}+T)+2 \ln \frac{\check{q}}{\widehat{q}}|1+\epsilon|-\gamma T<0, \\
\left(\frac{1}{b_{1}}, \frac{1}{b_{2}}, \ldots, \frac{1}{b_{M}}\right)^{T}>\widetilde{\Gamma}^{-1} \mathbf{1}_{M},
\end{gathered}
$$


where

$$
\begin{gathered}
\varphi=1+\theta+\gamma \check{q}+\frac{\check{b} \check{q}}{1-\bar{\tau}} e^{\gamma \tau}, \\
\widetilde{\Gamma}=\operatorname{diag}\left\{a_{1}, a_{2}, \ldots, a_{M}\right\}+\Gamma, \\
\check{a}=\max _{r \in S} a_{r}, \quad a_{r}=\frac{\lambda_{\max }\left(-2 \eta I_{n}+\check{p} \sum_{i=1}^{N} \Upsilon_{i 1}^{[r]}+2 \alpha_{r} P \Sigma\right)}{\check{p}}, \\
\check{b}=\max _{r \in S} b_{r}, \quad b_{r}=\frac{\lambda_{\max }\left(\sum_{i=1}^{N} P \Upsilon_{i 2}^{[r]}+2 \theta I_{N}\right)}{\widehat{p}},
\end{gathered}
$$

then the solutions $e_{1}(t), e_{2}(t), \ldots$, and $e_{N}(t)$ of system (9) are exponentially stable in mean square.

In another case, when we consider the system (3) without Markov switching, that is, $A^{[r(t)]}=A, B^{[r(t)]}=B$, and $\sigma_{i}^{[r(t)]}$ $\left(e(t), e(t-\tau(t)), e\left(t-\tau_{c}(t)\right)\right)=\sigma_{i}\left(e(t), e(t-\tau(t)), e\left(t-\tau_{c}(t)\right)\right)$, we can get another corollary.

Corollary 8. Let assumptions (H1) and (H2) be true and let $f \in \mathrm{QUAD}((P, \Delta, \eta, \theta)$. If there exist positive constants $\alpha$ and $\beta$ such that

$$
\begin{gathered}
{\left[\begin{array}{cc}
A+\check{\delta} I_{N}-\alpha I_{N} & \frac{B}{2} \\
\frac{B}{2} & -\beta I_{N}
\end{array}\right] \leq 0,} \\
\check{\tau} \leq \theta T, \quad \quad \check{\tau} \leq(1-\theta) T, \quad 0 \leq \check{\tau} \leq 1-\frac{b+c}{a}, \\
\varphi(\check{\tau}+T)+2 \ln \left|1+\epsilon_{v}\right|-\gamma T<0, \\
b+c<a,
\end{gathered}
$$

where

$$
\begin{gathered}
\varphi=a+\gamma+\frac{b}{1-\bar{\tau}} e^{\gamma \tau}+\frac{c}{1-\bar{\tau}_{c}} e^{\gamma \tau_{c}}, \\
a=\frac{\lambda_{\max }\left(-2 \eta I_{n}+\check{p} \sum_{i=1}^{N} \Upsilon_{i 1}+2 \alpha P \Sigma\right)}{\check{p}}, \\
b=\frac{\lambda_{\max }\left(\sum_{i=1}^{N} P \Upsilon_{i 2}+2 \theta I_{N}\right)}{\widehat{p}}, \\
c=\frac{\lambda_{\max }\left(\sum_{i=1}^{N} P \Upsilon_{i 3}+2 \beta_{r} P \Sigma\right)}{\widehat{p}},
\end{gathered}
$$

then the solutions $e_{1}(t), e_{2}(t), \ldots$, and $e_{N}(t)$ of system (9) are exponentially stable in mean square.

Furthermore, when we address the system (3) with fixed time delays, that is, $\tau(t)=\tau, \tau_{c}(t)=\tau_{c}$, the following corollary also can be obtained.
Corollary 9. Let assumptions (H1) and (H2) be true and let $f \in \operatorname{QUAD}(P, \Delta, \eta, \theta)$. If there exist positive constants $\alpha_{r}$ and $\beta_{r}$ such that

$$
\begin{gathered}
{\left[\begin{array}{cc}
A^{[r]}+\check{\delta} I_{N}-\alpha_{r} I_{N} & \frac{B^{[r]}}{2} \\
\frac{B^{[r]}}{2} & -\beta_{r} I_{N}
\end{array}\right] \leq 0, \quad \text { for } r=1,2, \ldots, M} \\
\check{\tau} \leq \theta T, \quad \check{\tau} \leq(1-\theta) T \\
\varphi(\check{\tau}+T)+2 \ln \frac{\check{q}}{\widehat{q}}\left|1+\epsilon_{v}\right|-\gamma T<0 \\
\left(\frac{1}{b_{1}+c_{1}}, \frac{1}{b_{2}+c_{2}}, \ldots, \frac{1}{b_{M}+c_{M}}\right)^{T}>\widetilde{\Gamma}^{-1} \mathbf{1}_{M}
\end{gathered}
$$

where

$$
\begin{gathered}
\varphi=1+\theta+\gamma \check{q}+\check{b} \check{q} e^{\gamma \tau}+\check{c} \check{q} e^{\gamma \tau_{c}}, \\
\widetilde{\Gamma}=\operatorname{diag}\left\{a_{1}, a_{2}, \ldots, a_{M}\right\}+\Gamma, \\
\check{a}=\max _{r \in S} a_{r}, \quad a_{r}=\frac{\lambda_{\max }\left(-2 \eta I_{n}+\check{p} \sum_{i=1}^{N} \Upsilon_{i 1}^{[r]}+2 \alpha_{r} P \Sigma\right)}{\check{p}}, \\
\check{b}=\max _{r \in S} b_{r}, \quad b_{r}=\frac{\lambda_{\max }\left(\sum_{i=1}^{N} P \Upsilon_{i 2}^{[r]}+2 \theta I_{N}\right)}{\widehat{p}}, \\
\check{c}=\max _{r \in S} c_{r}, \quad c_{r}=\frac{\lambda_{\max }\left(\sum_{i=1}^{N} P Y_{i 3}^{[r]}+2 \beta_{r} P \Sigma\right)}{\widehat{p}},
\end{gathered}
$$

then the solutions $e_{1}(t), e_{2}(t), \ldots$, and $e_{N}(t)$ of system (9) are exponentially stable in mean square.

Remark 10. In [29], the exponential stability of a class of stochastic neural networks with both Markovian jump parameters and mixed fixed time delays were investigated. Therefore, we could see our results as a further research about the stochastic dynamic network of [29].

\section{Numerical Simulation}

In this section, we present some numerical simulation results that validate the theorem in the previous section.

Consider the chaotic delayed neural network

$$
\begin{aligned}
d s(t)= & \{-C s(t)+A f(s(t))+B g(s(t-\tau(t)))\} d t \\
& +\sigma(s(t), s(t-\tau(t))) d w(t),
\end{aligned}
$$

where $f(s)=g(s)=\tanh (s), \tau(t)=1, \sigma(s(t), s(t-\tau(t)))=$ $\operatorname{diag}\left\{s_{1}(t), s_{2}(t)\right\}$,

$$
\begin{gathered}
C=\left[\begin{array}{ll}
1 & 0 \\
0 & 1
\end{array}\right], \quad A=\left[\begin{array}{cc}
2 & -0.1 \\
-5 & 4.5
\end{array}\right], \\
B=\left[\begin{array}{cc}
-1.5 & -0.1 \\
-0.2 & -4
\end{array}\right] .
\end{gathered}
$$




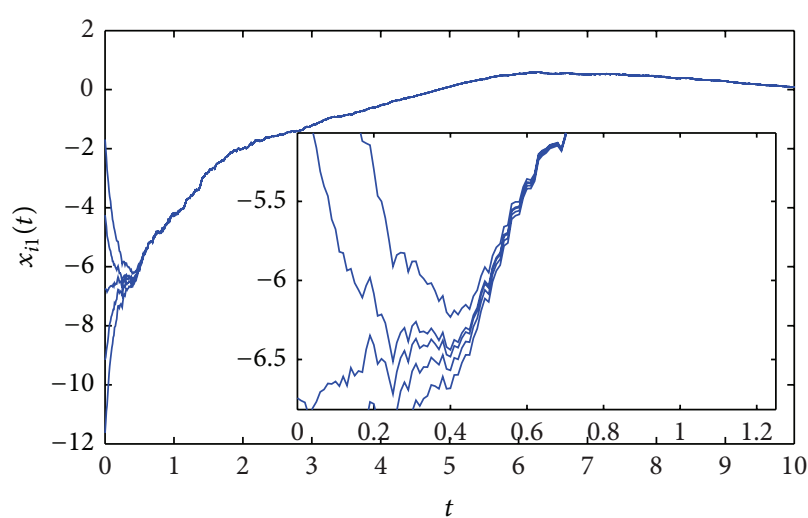

(a)

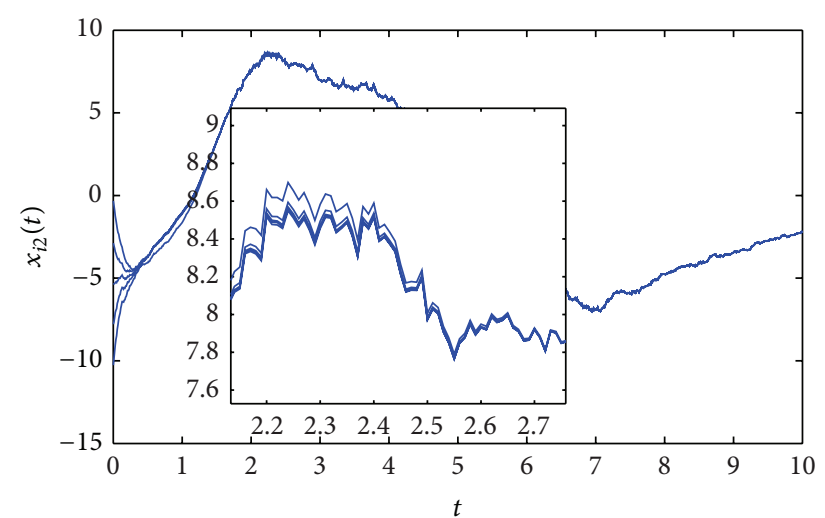

(b)

FIGURE 2: The trajectories of the state variables of $x_{i 1}$ and $x_{i 2}(i=1,2, \ldots, 5)$ in system (48) by impulse control.

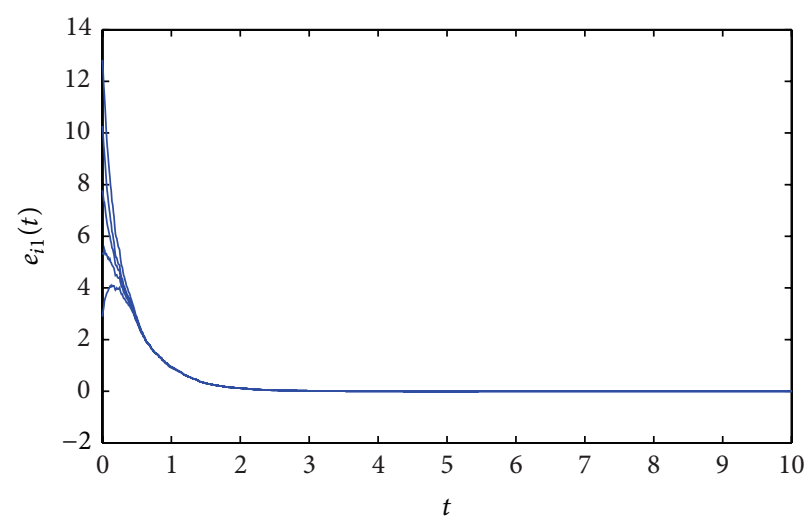

(a)

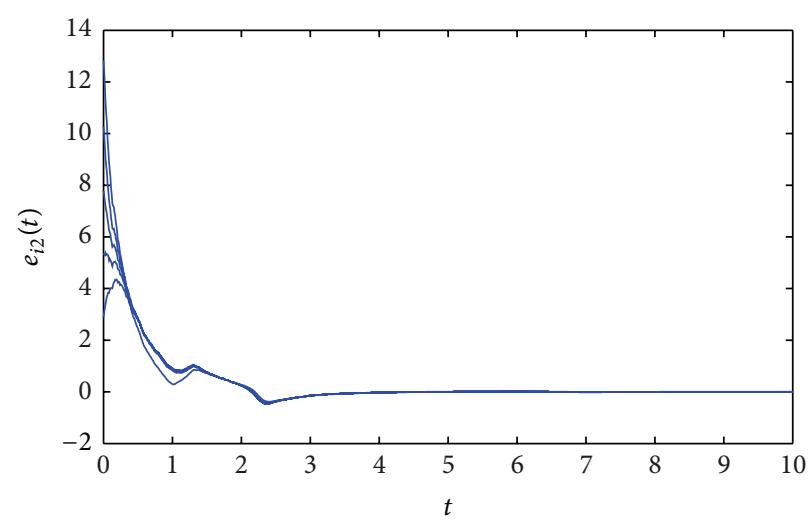

(b)

FIGURE 3: The time evolution of $e_{i 1}$ and $e_{i 2}(i=1,2, \ldots, 5)$ in system (48) by impulse control.

Taking $P=\operatorname{diag}\{1,2\}$ and $\Delta=\operatorname{diag}\{5,11,5\}$, we have $\eta=0.15$ and $\theta=3.25$ so that condition (11) is satisfied. Thus

$$
\begin{aligned}
& d x_{i}(t)=\left\{f\left(x_{i}(t), x_{i}(t-\tau(t))\right)+\sum_{j=1}^{5} a_{i j}^{[r]} \Sigma x_{j}(t)\right. \\
&\left.+\sum_{j=1}^{5} b_{i j}^{[r]} \Sigma x_{j}\left(t-\tau_{c}(t)\right)\right\} d t \\
&+\sigma_{i}^{[r]}\left(x(t), x(t-\tau(t)), x\left(t-\tau_{c}(t)\right)\right) d w_{i}(t), \\
& i=1,2, \ldots, 5, \quad r=1,2,
\end{aligned}
$$

and $\Gamma=\left[\begin{array}{cc}-3 & 3 \\ 2 & -2\end{array}\right], \tau_{c}(t)=0.1\left(e^{t} /\left(1+e^{t}\right)\right)$,

$$
\begin{aligned}
& \sigma_{i}^{[1]}\left(x(t), x(t-\tau(t)), x\left(t-\tau_{c}(t)\right)\right) \\
& =0.1 \operatorname{diag}\left\{x_{i 1}(t), x_{i 2}(t)\right\}, \\
& \sigma_{i}^{[2]}\left(x(t), x(t-\tau(t)), x\left(t-\tau_{c}(t)\right)\right) \\
& \quad=0.1 \operatorname{diag}\left\{x_{i 1}(t-\tau(t)), x_{i 2}(t-\tau(t))\right\} .
\end{aligned}
$$

Computations then yield $\tau=1, \bar{\tau}=0, \tau_{c}=0.1, \bar{\tau}_{c}=0.1$ and $Y_{i j}=0.1 I_{2}$ for $i=1,2, \ldots, N$. Then the solutions of inequalities (41)-(44) are (by using the Matlab LMI toolbox) $\alpha_{1}=2.500, \beta_{1}=0.001, a_{1}=4.305, b_{1}=5.055, c_{1}=0.109$; $\alpha_{2}=4.006, \beta_{2}=0.009, a_{2}=5.105, b_{2}=5.030$, and $c_{2}=0.065$.

The initial conditions for this simulation are $x_{i j}\left(t_{0}\right)$ which are constants, for $i=1,2, \ldots, 5, j=1,2$ and the trajectories of the impulse control gains are shown in Figure 2. Figure 3 shows the time evolution of the synchronization errors with impulse control.

\section{Conclusion}

In this paper, we investigated the synchronization problem for stochastic complex networks with Markovian switching and nondelayed and time-varying delayed hybrid couplings. We achieved synchronization by applying an impulse control scheme to a small fraction of the nodes and derived sufficient conditions for stability of synchronization. Finally, we considered some numerical examples that illustrate the theoretical analysis.

\section{Acknowledgments}

This work was supported by the National Science Foundation of China under Grant nos. 61070087 and 61273220, 
Guangdong Education University Industry Cooperation Projects (2009B090300355), and Shenzhen Basic Research Project (JC201006010743A and JCYJ20120613105730482). The authors are very grateful to the reviewers and the editor for their valuable comments and suggestions to improve the presentation of the paper.

\section{References}

[1] M. E. J. Newman, "The structure and function of complex networks," SIAM Review, vol. 45, no. 2, pp. 167-256, 2003.

[2] A. Arenas, A. Díaz-Guilera, J. Kurths, Y. Moreno, and C. Zhou, "Synchronization in complex networks," Physics Reports A, vol. 469, no. 3, pp. 93-153, 2008.

[3] R. Tönjes and B. Blasius, "Perturbation analysis of complete synchronization in networks of phase oscillators," Physical Review E, vol. 80, Article ID 026202, 7 pages, 2009.

[4] W. Lu, B. Liu, and T. Chen, "Cluster synchronization in networks of coupled nonidentical dynamical systems," Chaos, vol. 20, no. 1, Article ID 013120, 12 pages, 2010.

[5] J. Feng, J. Wang, C. Xu, and F. Austin, "Cluster synchronization of nonlinearly coupled complex networks via pinning control," Discrete Dynamics in Nature and Society, vol. 2011, Article ID 262349, 23 pages, 2011.

[6] J. Wang, J. Feng, C. Xu, and Y. Zhao, "Cluster synchronization of nonlinearly-coupled complex networks with nonidentical nodes and asymmetrical coupling matrix," Nonlinear Dynamics, vol. 67, no. 2, pp. 1635-1646, 2012.

[7] M. G. Rosenblum, A. S. Pikovsky, and J. Kurths, "Phase synchronization of chaotic oscillators," Physical Review Letters, vol. 76, no. 11, pp. 1804-1807, 1996.

[8] C. Van Vreeswijk, "Partial synchronization in populations of pulse-coupled oscillators," Physical Review E, vol. 54, no. 5, pp. 5522-5537, 1996.

[9] X. Yang and J. Cao, "Adaptive pinning synchronization of complex networks with stochastic perturbations," Discrete Dynamics in Nature and Society, vol. 2010, Article ID 416182, 21 pages, 2010.

[10] J. Lu, X. Wu, X. Han, and J. Lü, "Adaptive feedback synchronization of a unified chaotic system," Physics Letters A, vol. 329, no. 4-5, pp. 327-333, 2004.

[11] J. Wang, J. Feng, C. Xu, and Y. Zhao, "Exponential synchronization of stochastic perturbed complex networks with timevarying delays via periodically intermittent pinning," Communications in Nonlinear Science and Numerical Simulation, vol. 18, no. 11, pp. 3146-3157, 2013.

[12] Z. Li, X. Cao, and N. Ding, "Adaptive fuzzy control for synchronization of nonlinear teleoperators with stochastic time-varying communication delays," IEEE Transactions on Fuzzy Systems, vol. 19, no. 4, pp. 745-757, 2011.

[13] T. Yang, Impulsive Control Theory, vol. 272 of Lecture Notes in Control and Information Sciences, Springer, Berlin, Germany, 2001.

[14] G. Zhang, Z. Liu, and Z. Ma, "Synchronization of complex dynamical networks via impulsive control," Chaos, vol. 17, no. 4, Article ID 043126, 9 pages, 2007.

[15] T. Chen, X. Liu, and W. Lu, "Pinning complex networks by a single controller," IEEE Transactions on Circuits and Systems I, vol. 54, no. 6, pp. 1317-1326, 2007.

[16] J. Wang, C. Xu, J. Feng, M. K. Kwong, and F. Austin, "Meansquare exponential synchronization of Markovian switching stochastic complex networks with time-varying delays by pinning control," Abstract and Applied Analysis, vol. 2012, Article ID 298095, 18 pages, 2012.

[17] S. H. Strogatz and I. Stewart, "Coupled oscillators and biological synchronization," Scientific American, vol. 269, pp. 102-109, 1993.

[18] C. Gray, "Synchronous oscillations in neuronal systems: mechanisms and functions," Journal of Computational Neuroscience, vol. 1, no. 1-2, pp. 11-38, 1994.

[19] M. de Sousa Vieira, "Chaos and synchronized chaos in an earthquake model," Physical Review Letters, vol. 82, no. 1, pp. 201-204, 1999.

[20] R. Olfati-Saber, J. A. Fax, and R. M. Murray, "Consensus and cooperation in networked multi-agent systems," Proceedings of the IEEE, vol. 95, no. 1, pp. 215-233, 2007.

[21] O. Askari-Sichani and M. Jalili, "Large-scale global optimization through consensus of opinions over complex networks," Complex Adaptive Systems Modeling, vol. 1, no. 1, p. 11, 2013.

[22] J. Lu, D. W. C. Ho, and J. Kurths, "Consensus over directed static networks with arbitrary finite communication delays," Physical Review E, vol. 80, no. 6, Article ID 066121, 2009.

[23] X. Mao, "Stability of stochastic differential equations with Markovian switching," Stochastic Processes and their Applications, vol. 79, no. 1, pp. 45-67, 1999.

[24] C. Yuan and X. Mao, "Robust stability and controllability of stochastic differential delay equations with Markovian switching," Automatica, vol. 40, no. 3, pp. 343-354, 2004.

[25] Y. Sun and J. Cao, "Stabilization of stochastic delayed neural networks with Markovian switching," Asian Journal of Control, vol. 10, no. 3, pp. 327-340, 2008.

[26] Y. Liu, Z. Wang, and X. Liu, "On synchronization of discretetime markovian jumping stochastic complex networks with mode-dependent mixed time-delays," International Journal of Modern Physics B, vol. 23, no. 3, pp. 411-434, 2009.

[27] J. Lu, J. Kurths, J. Cao, N. Mahdavi, and C. Huang, "Synchronization control for nonlinear stochastic dynamical networks: pinning impulsive strategy," IEEE Transactions on Neural Networks and Learning Systems, vol. 23, no. 2, pp. 285-292, 2012.

[28] J. Zhou, L. Xiang, and Z. Liu, "Synchronization in complex delayed dynamical networks with impulsive effects," Physica A, vol. 384, no. 2, pp. 684-692, 2007.

[29] Q. Zhu and J. Cao, "Exponential stability of stochastic neural networks with both Markovian jump parameters and mixed time delays," IEEE Transactions on Systems, Man, and Cybernetics, Part B, vol. 41, no. 2, pp. 341-353, 2011. 


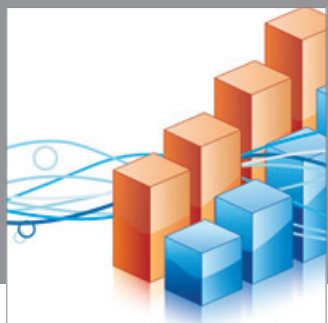

Advances in

Operations Research

mansans

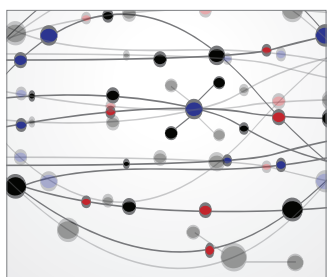

The Scientific World Journal
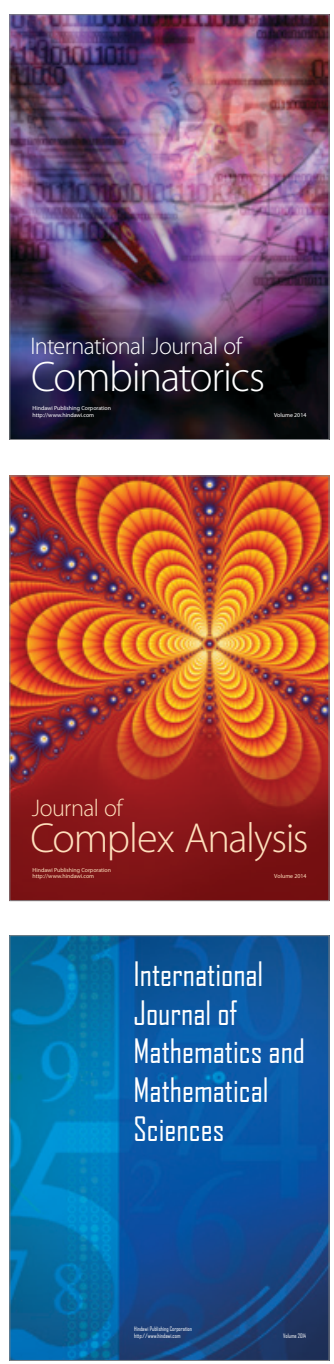
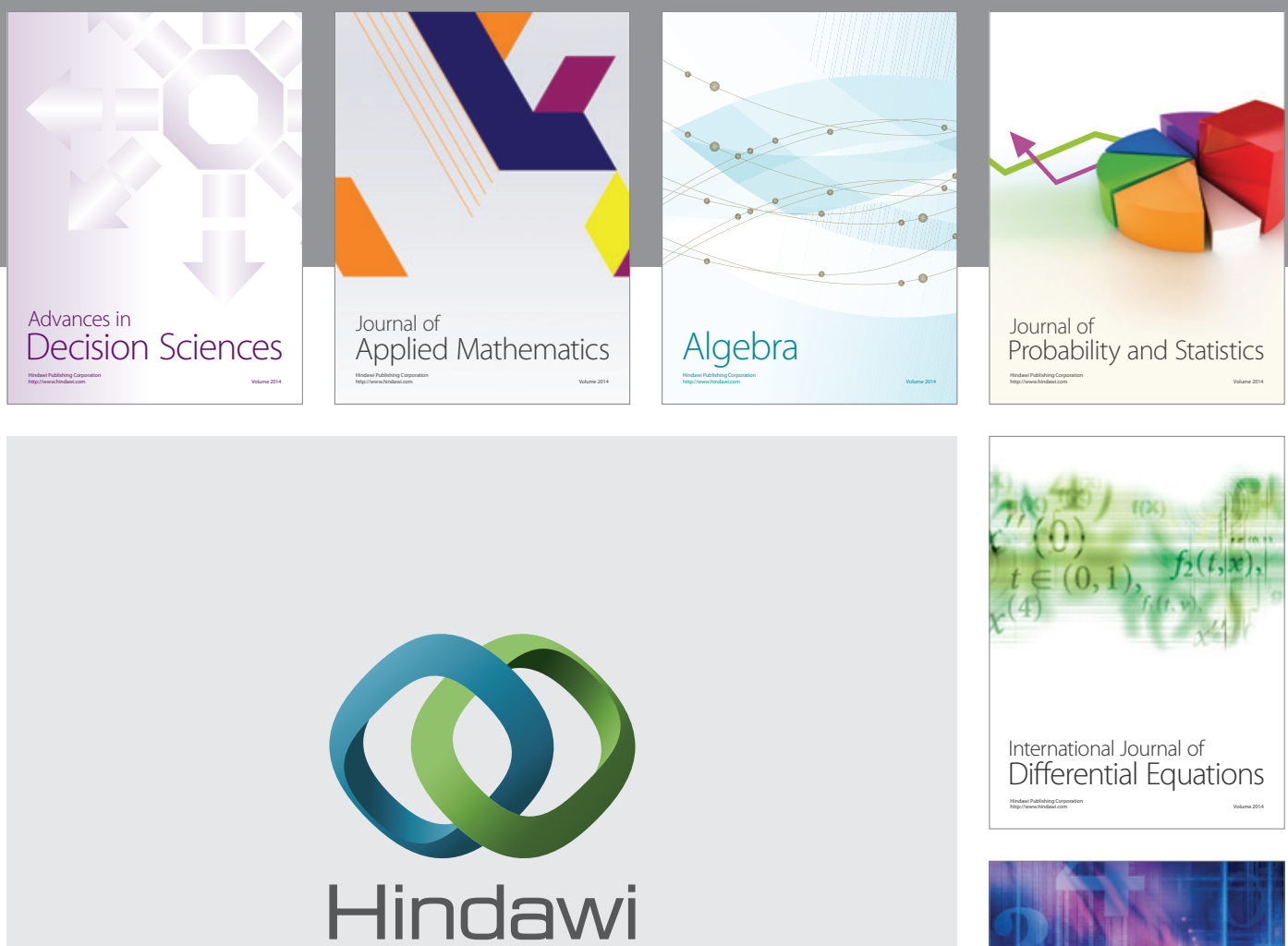

Submit your manuscripts at http://www.hindawi.com
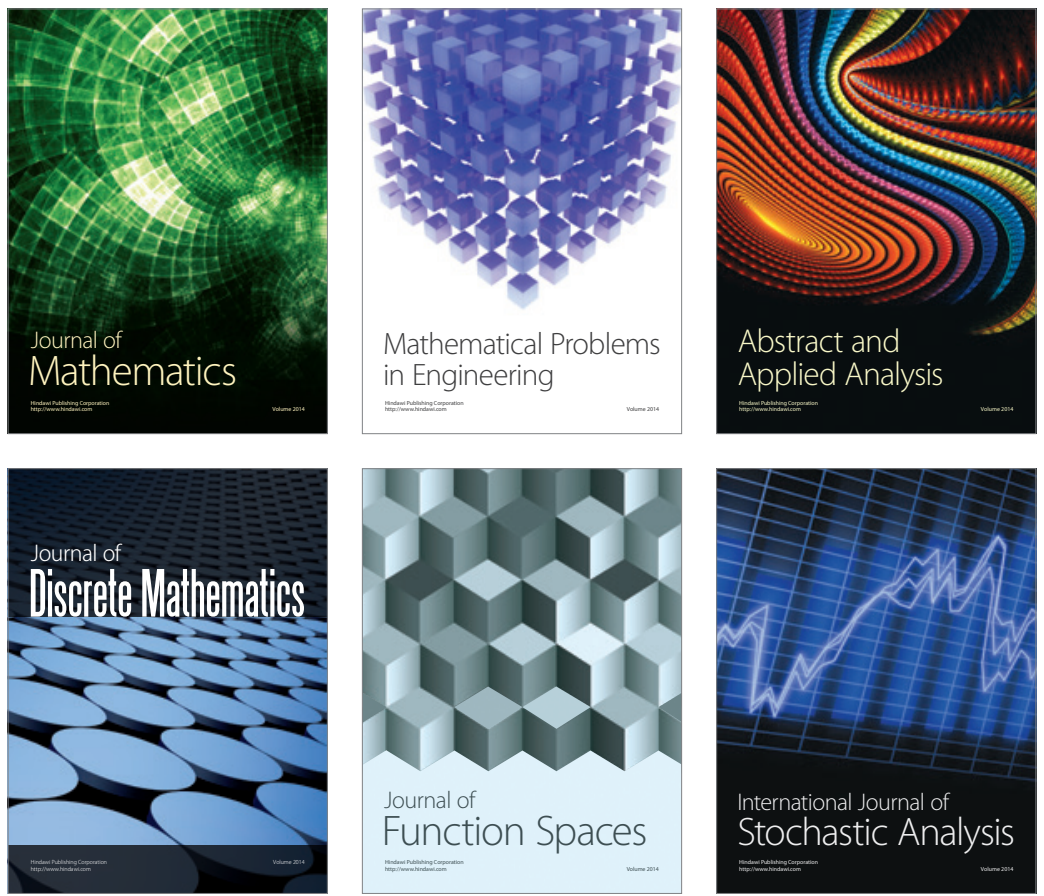

Journal of

Function Spaces

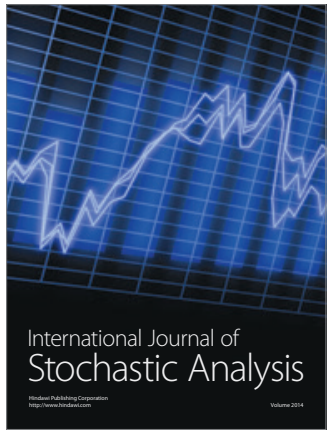

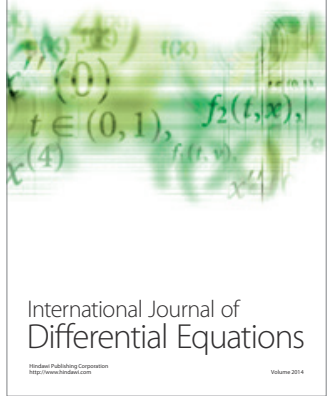
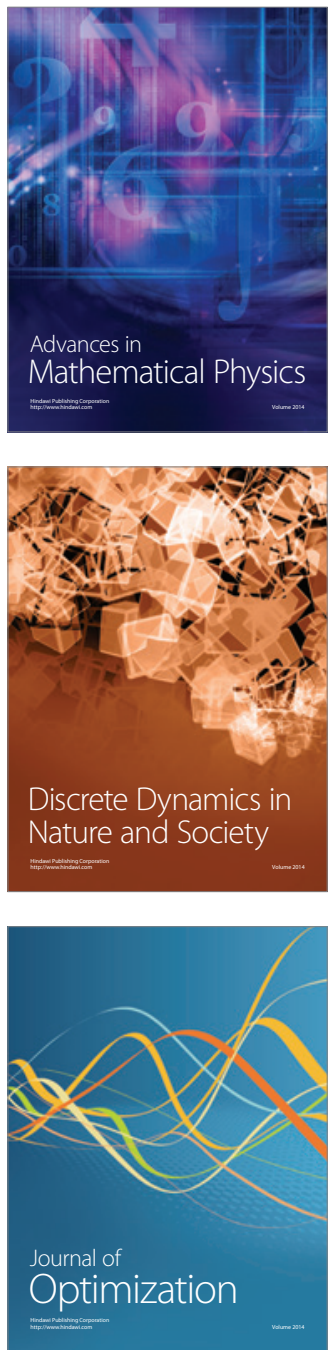